\title{
Los molokanos rusos de Baja California
}

\author{
Therese Adams Muranaka
}

\begin{abstract}
Resumen
La finalidad de esta investigación es aproximarse un poco más y averiguar quiénes son los molokanos y por qué huyeron de la Rusia imperial. Con este fin es necesario buscar las fuentes de la cristiandad ortodoxa. La separación de la iglesia católica y el catolicismo griego se dio en 1054, y seiscientos años después en 1654, sobreviene otra separación entre los ortodoxos rusos, de allí surgen los "creyentes a la antigua". En 1905, una colonia de agricultores rusos se estableció en el norte de Baja California, en el valle de Guadalupe, la cual ahora es conocida como Francisco Zarco o "colonia rusa". Aunque este artículo no llega a una conclusión para clasificar a este grupo, se espera que este estudio ilustre el parentesco étnico entre todos los colonos del valle. Se pueden diseñar algunas líneas susceptibles para su uso posterior en las que los lugares arqueológicos sugieren la etnicidad; aunque sin contar con la información histórica.
\end{abstract}

Palabras clave: molokanos, persecución religiosa, valle de Guadalupe, colonia Francisco Zarco, cristianos ortodoxos rusos, colonia rusa.

\begin{abstract}
The reason for this research is to find out who were the Molokans and the reasons why they escaped from Imperial Russia. To this end, it is necessary to search in the backgrounds of the Orthodox Christianity. Separation of the Catholic Church and Greek Catholicism was in 1054, and six hundred years later, in 1654, a new division inside Russian Orthodox Church took place. The result of this new division was the "Old fashioned Christian believers". In 1905, a colony of Russian farmers established in the North region of Baja California, in the Valle de Guadalupe, which currently is named Francisco Zarco or "Russian community". Even though this article does not reach to a conclusion as to classify this group, it is expected that this research offers insights to discover ethnical kinships amidst all the colonizers of the valley. In a future; regardless we do not have enough historical data, some susceptible lines of action could be designed to suggest certain archeological sites that will help to explain their ethnicity.
\end{abstract}

Keywords: Molokans, religious persecution, Valle de Guadalupe, Colonia Francisco Zarco, Russian Orthodox Christians, Russian community. 


\title{
LOS MOLOKANOS RUSOS DE BAJA CALIFORNIA*
}

\author{
Por \\ Therèse Adams Muranaka**

\begin{abstract}
En la antigüedad, el Antiguo y el Nuevo de un territorio a otro - como Abraham, Isaac, Jacob, Moisés y otros-, asi como las pruebas fehacientes de sus esfuerzos y
\end{abstract} \\ Testamento muestran los movimientos de gente \\ tribulaciones ante Dios (Shubin, 1963: 2).
}

Tanya Shubin miraba por la ventana mientras el tren hacía su recorrido a través de la selva de sofocante temperatura. Para una muchacha joven de las montañas de Armenia, el clima tropical de Panamá con aves y mamíferos desconocidos, resultaba intrigante y extraordinario, y tan sólo eran una muestra de las extrañas tierras que verían ella y su familia de agricultores rusos en tanto que construían su camino hacia la libertad. Cargando únicamente un pequeño maletín con las ropas sencillas que, como molokana profundamente religiosa, usaba; analizaba los sucesos que la habían llevado junto con su familia y otros molokanos a este recorrido al nuevo mundo, y se preguntaba hacia dónde sus fuertes convicciones religiosas los llevarían después.

Hace muchos años (1905), una colonia de agricultores rusos se estableció en la parte norte de Baja California, en el valle de Guadalupe, arriba de Ensenada. La colonia ahora conocida como Francisco Zarco o "colonia rusa", se localiza a 50 millas $(80.5 \mathrm{~km})$ al sur de Tijuana, ciudad que está ubicada en los límites internacionales. Constaba de 105 familias, las cuales unieron sus riquezas para comprar 10,000 acres de tierra $(4,000 \mathrm{ha})$ al gobierno mexicano, durante la presidencia de Porfirio Díaz. Las escrituras oficiales son de 1907; sin embargo, los primeros colonos recuerdan una colonia establecida en ese lugar antes de esa fecha (Deway, 1966: 36 y el apéndice A Diario San Diego Unión del 26 de agosto y del 5 de septiembre de 1905).

Esta gente fue conocida como los molokanos, y en el informe del escritor ruso León Tostoi (Young, 1932; Dunn, 1967: 307) se señala que llegaron al Nuevo Mundo familia por familia desde los alrededores de Kars, actualmente Turquía. Así como muchos grupos de inmigrantes, ellos llegaron a América con un propósito: eran miembros de un grupo religioso cristiano que creía en la paz, la Biblia y en la vida sencilla. En sus esfuerzos por evadir la intromisión del gobierno imperial ruso en su modo de vida privada (Rogoff, M. 1983; Conybeare, 1962: 293-294), algunas familias huyeron a Ucrania en Rusia; a Bremen o

\footnotetext{
* Traducido del inglés por Guadalupe Ortega Villa.

** Este artículo forma parte de su tesis para obtener el grado de Doctor en Antropología.
} 
Hamburgo, y desde allí a la isla Ellis; Galveston, Texas; o por tren a través del istmo de Panamá (ya que el canal no fue abierto antes de 1914). Algunos llegaron a Estados Unidos vía Harbin, en China; otros llegaron después de un momento de calma en Argentina, Chiley otros países latinoamericanos (Lisizin, 1984: 11).

Sufriendo muchas penalidades, llegaron juntos a Los Angeles, California a principios de 1904 (Desatoff, 1977; Deway, 1966: 33; Lisizin, 1984: 10; Young, 1927: 54). Las familias asentadas en la "aldea rusa", se convertirían en "los descastados"de Boyle Heights o de Hollenbeck Heights, Belvedere, Maywood, Bell, Huntington Park, San Pedro, Lynwood y Palomar Park (Dolbee, 1983; Sokoloff, 1918; Young, 1932: 16). Los molokanos recuerdan que sabían que Los Angeles era su lugar de destino, porque se les profetizó que ellos siempre estarían protegidos por "los ángeles". Encontrando que las tierras eran caras, y que necesitaban grandes terrenos para cultivar el trigo de invierno a la usanza antigua (Schmieder, 1928: 415-420), empezaron de nuevo a buscar unas más baratas así como un estilo de vida menos materialista. Oficialmente adquirieron en 1907 un terreno grande en México conocido como la ex misión de Nuestra Señora de Guadalupe, antiguamente una misión dominica en el valle de Guadalupe arriba de Ensenada (Goldbaum, 1971: 56), asentándose allí con el propósito de mantener por siempre sus costumbres antiguas (figura 1).

Los molokanos construyeron viviendas de adobe pintadas de blanco, una tras otra a lo largo de un único camino recto con una hilera de árboles a los lados (modelo Strassendorf). Las casas estaban hechas de barro secado al sol y ladrillos de adobe mezclados con paja al estilo de los adobes mexicanos de Baja California, pero con techos cubiertos de teja con un grado de inclinación a la manera rusa para mantener fuera la nieve. Cada familia tenía su faja de tierra del mismo tamaño sobre la calle principal,y la entrada de cada casa era a través de un estrecho pasillocon un cuarto a cada lado, uno de los cualesera la cocina. Tal vez en la parte de atrás existía otra cocina anexa, con un horno para cocinar o pechka (Story, 1960: 35), un baño de vapor, conocido como banya, consistente de un piso de piedras que calentaban y en las cuales se vertía rápidamente agua fría funcionando como un sauna para toda la familia, recordando los agasajos de los sábados por la noche para los primos visitantes de Los Angeles (Deway 1966: 49). Fuera de la casa, un cuarto para lavar, un gallinero (quizácon un cuarto arriba para los jornaleros), un corral para patos y un granero, completaban la mayoría de las casas (Story, 1960: 36), las cuales tenían un pequeño jardín, así como quizá también un molino de viento.

Los interiores de las viviendas han variadoconsiderablemente a lo largo de la historia de la colonia. Los dos cuartos, la cocina y el comedor, tienen camas con colchones de pluma y enormes cestos para almacenar, que en 1960 aún contenían cobertores fabricados en Rusia. Sin embargo, las sábanas eran hechas en México o en Estados Unidos (Story, 1960: 36). Estufas de madera y últimamente de gas, bancas construidas en las paredes, recipientes para mezclar, ollas para el café, vasos chicos para el té y salseras chinas, cucharas y recipientes de madera, todo esto amueblaba los interiores(Rogoff,1988). Enexhibición y siempre 


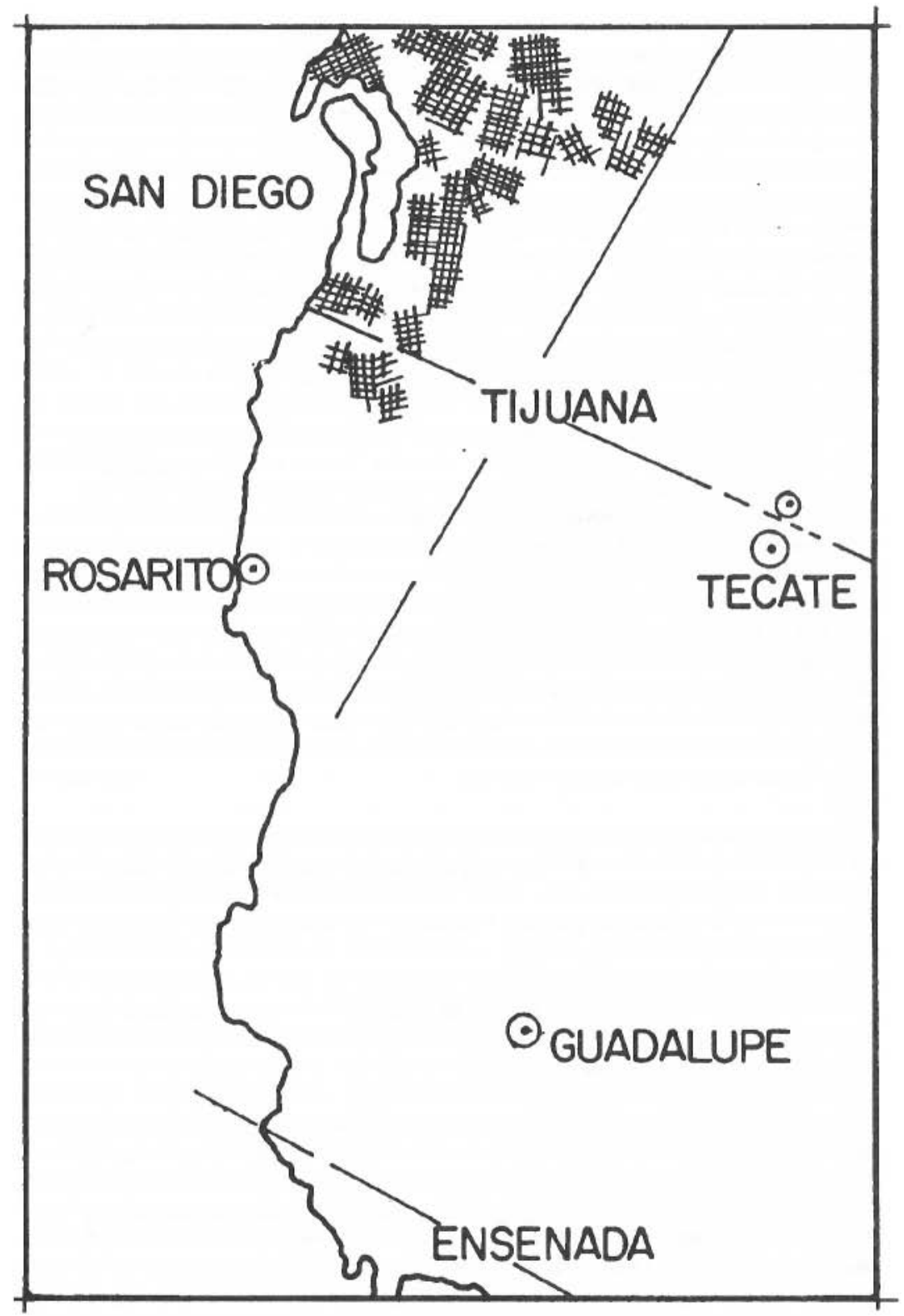

FIGURA 1. Valle de Guadalupe, Baja California. 
-sentes, el servicio de samovar o té ruso, y una Biblia; fotos de bodas, quizá una estampa a colores de la Ultima cena y posiblemente con el tiempo se permitió el uso del fonógrafo (Story, 1960: 38; Patzakayo, 1980). Los pórticos estaban cubiertos de vigas de madera labrada, diferentes de las casas mexicanas.

En la villa no existía iglesia elaborada, sino sólo una sencilla, de edificio simple sin iconos o estatuas, y entre finales de 1955 a 1957 se construyó un templo nuevo (Story, 1960: 60, 127). Los servicios de este sobranie, o lugar de reunión, eran llevados a cabo cada semana con diferente horario y consistían en cantos líricos y oraciones que invocaban la influencia del Espíritu Santo en los pensamientos y discursos de cualesquier persona que debiera hablar. Debido al fervor de las reuniones, la gente era-conocida como "los de espírituexaltado". El pacifismo, la sobriedad y la conciencia social eran alabados.

Está en contra de nuestra religión que un hombre se considere mejor que sus semejantes en cualquier sentido. Todos somos iguales ante Dios. La única superioridad que un hombre mantiene sobre otro está en sus años de experiencia y sabiduría espiritual. (Palabras de un anciano citado por Young, 1928: 546).

De acuerdo con estos puntos de vista, la indumentaria de los molokanos era sencilla. Un hombre ruso vestía una blusa al estilo cosaco con un cordón entrelazado como cinto, pantalón bombacho largo y botas. Las mujeres vestían faldas largas, sobreblusas y en ocasiones especiales usaban en la cabeza chales de hilo tejido a mano, conocidos comokosinkas. Las mujeres casadas se ataban el cabello a la altura del cuello con listones. No se permitia el uso de adornos o joyería que no tuviera alguna función.

Tal y como lo dictaba su religión, todas las cosechas eran obtenidas con la participación de todos y los alimentos eran almacenados y distribuidos por los ancianos designados para ello. Todo era fabricado en casa individualmente, excepto el café, azúcar, sal y arroz, los cuales eran adquiridos en Ensenada o en San Diego (Rogoff, 1983).

Diariamente la comida era preparada sencillamente, y la carne rusa borscht, lapscha o fideos, y las hogazas de pan de trigo eran cocidos en los hornos ubicados fuera de las casas.

Los molokanos introdujeron los gansos y la apicultura a Baja California. Ellos no consumían alcohol, mariscos o animales de pezuña, por ejemplo el cerdo ("El cerdo no mira al cielo"). Después, el chivo y el borrego fueron considerados como comida para los invitados, junto con los alimentos procedentes de sus huertos, tales como coles curtidas, pepinos, aceitunas, cebollas, calabazas y melones (Post y Lutz, 1976: 144, Story, 1960: 31).

Molokano, nombre asignado a las personas pertenecientes a esta secta, es similar a la palabra rusa "leche" (moloko). Los estudiosos de las sectas rusas, como Klibanov (1982: 107-109), no dejan duda de que "río lácteo" (molochnye 
vody) proviene de los doukhobors, de quienes los molokanos evolucionaron, pero la mayoría dudan de la vinculación entre "río lácteo" y el nombre molokano (Dunn y Dunn, 1967: 352). Una segunda explicación de su nombre es aquélla que surge a principios de la persecución de la secta en Rusia, cuando la leche y los productos lácteos eran la única comida permitida por los molokanos de conciencia impugnadora, aprisionados por sus creencias, y era la única de la que podían estar seguros de no estar contaminada con grasa de cerdo, de tal forma que los molokanos siguieron las antiguas leyes judías (Samarin, 1988). Existen muchas referencias acerca del hecho de que el término "molokano" proviene de su hábito de beber leche durante las vigilias ortodoxas rusas - como el tiempo de cuaresma-, períodos durante los cuales a los ortodoxos no les era permitido ingerir leche o productos lácteos (Lane, 1978: 100; Shubin, 1963: 10; Struve, 1967:228). La explicación más comúnmentedada actualmente en el valle de Guadalupe es que el nombre viene de la primera epístola de Pablo a los corintios, capítulo 3, versículo 2, acerca de los cristianos bebiendo "leche espiritual".

El propósito de aproximarnos un poco más de cerca y averiguar quiénes son los molokanos y por qué huyeron de la Rusia imperial, nos lleva a indagar en los orígenes de la cristiandad ortodoxa. En el año de 1054 d. C., el catolicismo latino y el catolicismo griego (después conocido como ortodoxo) se separaron bajo el liderazgo de Miguel Cerularis, y seiscientos años después, en 1654, se dio la separación de los ortodoxos rusos. La tercera roma de nuevo fue dividida por la reacción de las protestas en contra de los cambios de ritos hechos por el patriarca Nikon. Estos inconformes fueron conocidos como los "creyentes a la antigua”, y desde el punto de vista ortodoxo, eran cismáticos peligrosos, o raskolniki.

... los campesinos ortodoxos decían que entre los raskolniki cada hombre moujik (campesino) formaba una secta, cada baba (mujer anciana) un credo (Stepniak, 1888: 266).

Separándose, juntándose y volviéndose a separar, los rascolniki básicamente se dividieron en dos sectas: los popovtsy (aquéllos con sacerdotes) y los bezpopovtsy (aquéllos sin sacerdotes). Los molokanos son una secta sin sacerdotes formada aproximadamente en 1765 a partir de una separación de los doukhobors rusos, un grupo ahora famoso en el oeste de Canadá (Kolarz, 1961: 349; Stepniak, 1888: 266; Struve, 1967: 228), también conocidos como "cristianos espirituales". Su fundador fue un sastre ruso que se llamaba Simón Uklein, cuya doctrina (escrita en un libro llamado El ritual) incluía los siguientes preceptos: no sacerdotes, no organización formal de la iglesia, no diferencias de status y una oposición al progreso material (Young, 1932: 83-84). El bratsvoo "hermandad"y el obshchestvo o "comunidad"eran sus ideas centrales. Sin embargo, se diferencian de los doukhobors, quienes casi sólo eran campesinos, en que los molokanos pronto procuraron hacer prosélitos de la gente de la población (meshchanye), comerciantes e industriales (Lane, 1978: 100). El pacifismo, la razón y el autoperfeccionamiento por medio del trabajo eran altamente valorados (Lane, 1978: 102). Los molokanos han sido caracterizados, sobre todo, como 
una secta utópica que busca reconstruir el mundo con un mejor estilo de vida, pero distante de sociedades más grandes (Wilson, 1970: 47). Los molokanos obran como secta, sociológicamente hablando; en eso se diferencian del mundo exterior. Inspirados por la divinidad, se autoapartan por medio de su vestimenta sencilla y de su discurso; además, tienen todo un cuerpo de leyes codificado, que consideran sagrado (Young, 1932: 56-57).

La secta se extendió con Uklaine desde Tambov a Veronezh, a Mihailovsky, a los cosacos del río Don, a Saratov, y de allí al Cáucaso con Isaias Ivanov Krylov, y a través del río Volga con Pedro Dementev (Conybeare, 1962: 291). Una expansión más amplia es mencionada por otros autores, y hacia la región de Ryazan por un seguidor llamado Moisés El Dálmata.

Pronto surgieron las divisiones doctrinarias en la secta (Young, 1932: 71); los que se separaron del grupo molokano fueron los subbotniki o "sabatarios" (quienes siguieron las leyes judías más apegadamente), los "cristianos evangélicos" del río Don (que terminaron por ser asimilados), los "comunistas" (quienes hicieron varios esfuerzos por lograr la igualdad completa a través del reparto de sus propiedades), los postoyanyi o "inmutables" (quienes no "brincan" en éxtasis con el Espíritu Santo en las reuniones religiosas), y los pryguny o "de espíritu exaltado" (también llamados maksimisty en honor a Máximo Rudametkin) (Lane, 1978: 102).

La persecución religiosa se iniciaba cada vez que los asentamientos de los desplazados molokanos crecían en prosperidad o, a excepción de los cristianos evangélicos, cuando el gobierno imperial ruso iba a la guerra o necesitaba soldados, a lo cual los molokanos se negaban (Rogoff, M. 1983). Fue así como durante esta época se decretaron leyes severas en contra de los molokanos (Conybeare, 1962: 293-294). En respuesta a estos nuevos problemas y a instancias del famoso escritor y pacifista ruso León Tolstoi, los molokanos iniciaron entre 1904 y 1906 su desorganizada salida de Rusia, familia por familia (Dunn y Dunn, 1967: 307; Tolstoi, 1933).

Huyeron en todas direcciones; aproximadamente 10,000 personas emigraron a los Estados Unidos saliendo vía Hamburgo o Bremen y entrando por la isla Ellis; por Galveston, Texas; o a través del istmo de Panamá(Young, 1932: 14). Casi todos los molokanos encontraron en 1904 la forma para llegar al centro de Los Angeles, California, siguiendo a los líderes Felipe M. Shubin e Iván Samarín, quienes en 1900 llegaron como avanzada para hacer exploraciones en busca de tierra (visitando a los doukhobors de Canadá y trabajando durante sus viajes a San Francisco y Los Angeles) (Shubin, 1963: 26). Las familias molokanas se asentaron en los suburbios de Los Angeles ya mencionados (Moore, 1973: 21-22), donde el corazón del asentamiento molokano eran las calles Vignes y Primera, sector en donde se localizaba la tienda de los Rudametkin. Sus negocios eran identificables por los nombres terminados con los sufijos: -in, -off, o -eff.

Los acontecimientos de la modernidad no han sido sencillos para los molokanos del valle de Guadalupe. En una colina en la cual se puede observar toda la 
colonia, el cementerio cuenta la historia. En la cima del cerro aún se pueden observar las hileras de las lápidas más antiguas, escritas en alfabeto cirílico con traducciones al español, por ejemplo: Sergei Filatoff a "Sergio Filatoff". A los pies del cerro hay lápidas escritas solamente en español decoradas con muchos detalles como flores, cruces y figuras religiosas. Estas piedras son pruebas fehacientes de la muerte, de los matrimonios con personas ajenas a la secta y del reciente abandono de la villa.

La primera emigración importante de la cual se desconoce el total de colonos, se llevó a cabo en 1912 (Schmieder, 1928: 421), y quizá fue motivada por estar los colonos insatisfechos con la tierra o tal vez por el temor del incipiente movimiento revolucionario en el norte de México (cf. "Los pacíficos" en Reed, 1983: 57); ciertamente el folklore popular menciona más de un disturbio provocado por los villistas en busca de comida, ropa y caballos (Rogoff, 1983; Samarín 1988).

El segundo gran éxodo del valle se efectuó después de que se formó el ejido "El Porvenir" en 1937. La Revolución Mexicana pedía "Tierra y Libertad". El artículo 127 de la Constitución Política de los Estados Unidos Mexicanos dice:

La propiedad de las tierras y las aguas ... corresponde originariamente a la nación la cual . . . tiene el derecho de transmitir el dominio de ellas a los particulares ... La nación tendrá en todo tiempo el derecho de imponer a la propiedad privada las modalidades que dicte el interés público ... . con objeto de hacer una distribución equitativa de la riqueza pública... (Deway, 1966: 52).

Para acatar el Código Agrario de México, estas leyes le dieron el derecho al presidente Lázaro Cárdenas para repartir porciones de tierra a la población en general, procedimiento conocido como sistema ejidal (Meyer y Sherman, 1979: 378).

El 19 de septiembre de 1937, el gobierno mexicano solicitó la dotación o “donación", y el 28 de noviembre de ese mismo año 2,920 hectáreas cercanas a la colonia rusa fueron repartidas a 58 ejidatarios mexicanos. El ejido conocido como "El Porvenir" no se localizó en las tierras rusas, pero de igual forma el reparto provocó gran consternación entre los rusos. Colonizadores como María Rogoff (1983) invitaron al presidente Cárdenas para que visitara la colonia, quien impresionado por sus granjas tan industriosas, decidió no tomar las tierras rusas para ampliar el ejido. Un sinnúmero de colonos no quedaron convencidos y estrecharon sus lazos en Estados Unidos.

El último cambio en la colonia se presentó cuando en 1958 se construyó un camino nuevo (Deway, 1966: 82; Story, 1960: 162; Kvammen, 1976) y cuando llegaron los advenedizos, quienes reclamaron porciones del valle como de su propiedad. El año de 1958, en particular, fue un año de elecciones, y los activistas de la Unión General Obrera y Campesina (UGOCM) organizaron las tomas de posesión de terrenos privados para acelerar la reforma agraria (Deway, 1966: 80). Asociados a este movimiento, 3,000 trabajadores aparecieron en el 
valle de Guadalupe en el transcurso de la noche del 10 de julio de 1958; llegaron como "paracaidistas" por su repentina presencia "de los cielos", y formaron el poblado Francisco Zarco - en memoriadel personaje políticoliberal mexicano(cf. Meyer y Sherman, 1979: 385-386), en el lugar donde se junta la calle principal y la carretera Tecate-Ensenada. Respaldados por la "Ley de Tierras Ociosas", el 23 de junio de 1920 los invasores fueron atraídos por las tierras rusas abandonadas.

A esto le siguieron actos de desobediencia civil mientras más y más intrusos llegaban. La destrucción de plantíos, asaltos a los almacenes, robos en los huertos, fueron algunas de las técnicas usadas para presionar a los rusos para que dejaran sus tierras. Muñecas y osos de juguete fueron incendiados y se amenazó a la gente con quemar sus cosechas (Deway, 1966: 84; Rogoff, 1983). En agosto de 1959, 107 hectáreas fueron oficialmente otorgadas al poblado. La preocupación por sus pertenencias, las cuales no eran constantemente vigiladas, los forzó a vender más y más parcelas, llevando a la muerte el funcionamiento de la colonia.

Con el paso del tiempo, colonos japoneses, chinos y judíos, entre otros grupos étnicos, compraron propiedades a los intrusos, cambiando por completo las características étnicas de la villa. Actualmente sólo existe una familia de "rusos puros" en la colonia y seis o siete hombres rusos casados con mexicanas. Los cosacos rusos de Ensenada han vivido en el valle, y debido a su lenguaje, han dejado excelentes memorias en la historia de la zona. (cf. Lisizin, 1984).

Actualmente todo lo que queda es una colonia en ruinas. Los residentes denuncian que en diez años más ningún ruso será escuchadoen el valle, el cual era conocido por el sonido de las voces rusas en la capilla, a medida que los visitantes se aproximaban al camino principal (Samaduroff, 1983; Long, 1983). Los visitantes de hoy en día no ven nada de la villa en donde Tanya Shubin - la muchacha que cruzó el istmo de Panamá-vivió con su esposo Moisés Desatoff. Sus hijos, nietos y bisnietos viven al norte, en California (Dolbee, 1983). Nadie en la villa recuerda cuando se sentaba tranquila, cargando a su pequeña hija en brazos, mientrasse dirigía a la frontera norteamericana, que cruzaba para sepultar a la niña en Los Angeles, en un lugar más familiar. Muchos sufrimientos, muchas alegrias asociados a los rusos de Guadalupe han sido olvidados. Hoy, al caminar por el valle de Guadalupe, puede uno darse cuenta de que está en ruinas, llenode maleza. El visitante ocasional, quizá un arqueólogo, que mire hacia lo largo del camino, verá Salsola kali o amaranto, también conocido como cardo ruso.

Porque vos no habéis recibido el espíritu del cautiverio de nuevo para temer, sino habéis recibido el Espíritu de Adopción, por lo cual clamamos Abba, Padre. El mismo Espíritu certifica con nuestro espíritu que somos hijos de Dios, y si somos hijos, entonces somos herederos, herederos de Dios y coherederos con Cristo; sí es así que sufrimos con El para que podamos ser glorificados juntos. Porque reconozco que los sufrimientos presentes no son dignos de ser comparados con la gloria que nos será revelada (Shubin, 1963). 
Un lugar como Guadalupe necesita de un arqueólogo. Después de que uno ha entrevistado a los seis o siete rusos que aún permanecen allí, los recuerdos truncos que quedan en su débil memoria, hacen reconocer que es necesario un medio mejor para investigar. Se solicitóal Instituto Nacional de Antropología e Historia (INAH) un permiso para hacer excavaciones e investigar el pasado del valle de Guadalupe desde el período ruso (1905) a la fecha. El permiso fue otorgado en 1985 tanto por el INAH como por el Departamentode Antropología de la Universidad de Arizona en Tucson, y la excavación seinició en octubre de ese año. Las cuadrillas estaban compuestas por trabajadores mexicanos y norteamericanos. Las pruebas tuvieron lugar en las casas de las personas que tienen ascendencia rusa pura hasta la actualidad, así comoen aquéllas de los que se casaron con mexicanos. Los exámenes se aplicaron también en las casas que habían pertenecido a rusos y que fueron vendidas a mexicanos sin herencia rusa.

Tal y como se prometió, la misión de Guadalupe y varios sitios kumiai no fueron alterados.

Encuestas, mapas, fotografías y pruebas de excavaciones fueron efectuadas bajo técnicas internacionalmente aceptadas para investigaciones científicas. Con la supervisión de Julia Bendímez del Centro Regional del INAH y de David Zárate Loperena, del INAH de Ensenada (en cuya casa se guardaban todos los instrumentos), se descubrieron aproximadamente 4,000 objetos de relativa antigüedad. La mayoría de ellos consistían en botellas rotas, latas, partes de autos, equipo agrícola, herraduras, botones, cierres, piezas rotas de porcelana china, pedazos de huesos de animales. Se descubrieron dos utensilios kumiai, ambos de $5 \mathrm{~cm}^{2}$. Uno era un pedazo de roca volcánica usado en la preparación de herramientas de piedra, y el otro era un pedazo de cerámica. No se encontró objeto alguno perteneciente al período español.

El diseño de la investigación consistió en preguntas de tipo étnico en cuanto al parentesco de los rusos del valle con sus vecinos mexicanos e indígenas. Partiendo de que un grupo solo no puede ser étnico, la perspectiva de los estudios étnicos es la comparación entre un grupo y otro. Desde una perspectiva interna, su etnicidad consiste en su percepción de la distancia social entre sí mismo y los miembros de otros grupos sociales; tal como se siente un ruso en relación a los mexicanos que viven alrededor suyo (Portes, 1986: 383). Se asume que su percepción se representa en su comportamiento; los patrones de conducta como los hábitos alimenticios por ejemplo - reflejados en artículos y utensilios de cocina, serán significativamente diferentes y con significado estadísticamente definido. La arquitectura, la sencillez en el estilo de los objetos para reflejar la motivación religiosa de la colonia rusa y la semejanza de ellos para manifestar la cohesión interna de la colonia, se encuentran entre los patrones que fueron revisados. A la fecha de este reporte preliminar no se habían diseñado conclusiones hasta no terminar con la clasificación. La esperanza es que el estudio ilustrará el parentesco étnico entre todos los colonos del valle en este caso que se ha tratado de documentar. La explicación es que pueden diseñarse algunas líneas susceptibles de ser usadas en otras ocasiones, en las que los lugares arqueológicos sugieren la etnicidad, pero sin tener información histórica. 


\section{BIBLIOGRAFIA}

CONYBEARE, Frederick. 1962. Russian Dissenters. Russell and Russell, Inc., New York.

DESATOFF, Tanya. 1977. Entrevista grabada, Woodburn, Oregon. Abril 24, 1977.

DEWAY, John Stanford. 1966. The Colonia Rusa of Guadalupe Settlemant, Competition and Change. M.A. Geography, California State University, Los Angeles.

DOLBEE, Judie. 1983. Comunicación personal.

DUNN, Ethel. 1967. "Canadian and Soviet Doukhobors: An Explanation of the Mechanisms of Social Change". Canadian Slavic Studies IV: 2 (Summer).

DUNN, Stephen P. y Ethel Dunn. 1967. The Peasants of Central Russia. Holt, Rinehart and Winston, New York.

FADNER, F.L. 1967. "Russian Sects". In: New Catholic Encyclopedia. Vol. XII.

GOLDBAUM, David. 1971. Towns of Baja California: A 1918 Report. Translated with introduction and supplemental annotions by William $\mathrm{O}$. Hendricks. La Siesta Press, Glendale, California.

KLIBANOV, A. I. 1982. History of Religious Sectarism in Russia (1860's1917), edited by Stephen P. Dunn, translated by Ethel Dunn. Pergamon Press.

KOLARZ, Walter. 1961. Religion in the Soviet Union. MacMillan: London, St. Martin's Press, New York.

KVAMMEN, Lorna. 1976. A Study of the Relationship Between Population Growth and the Development of Agriculture in the Guadalupe Valley, Baja California, México. Tesis de maestría en antropología, California State University, Los Angeles.

LANE, Christel. 1978. Christian Religion in the Soviet Union: A Sociological Study. George Allen and Unwin, London.

LISIZIN, Francisco. 1983. Secta religiosa molokan y la colonia rusa de Guadalupe, Ensenada, Baja California, mimeo.

LONG, Helen. 1984. Comunicación personal.

MALONEY, G. A. y A. Wuyts. 1967. "Russian Rite". In: New Catholic Encyclopedia, vol. XII McGraw-Hill Book Company.

MEYER, Michael C. y William L. Sherman. 1979. The Course of Mexican History. Oxford University Press. New York.

MOORE, Willard Burgess. 1973. "Molokan Oral Tradition: Legends and Memorates of an Ethnic Sect". University of California Publications. Folklore Studies № 28.

PALMIERI, A. 1913. "The Religion of Russia". In: Catholic Encyclopedia, edited by Charles G. Hebermann, vol. XIII. The Encyclopedia Press, New York.

PATZAKAYO, Susana. 1980. Comunicación personal citada en Robert Montemayor, "Baja's Russian Colony Dwindling Away", Los Angeles Times, de febrero 10 de 1980, sección 2, p. 10.

PORTES, Alejandro. 1986. "The Rise of Ethnicity: Determinants of Ethnic Perceptions Among Cuban Exiles in Miami”. American Sociological Review, 49 (3). 
POST, Lauren C. y Carl Lutz. 1976. The Molokan Russian Colony of Guadalupe, Baja California, México. Brand Book IV. San Diego Corral of the Westerners.

REED, John. 1983. Insurgent Mexico. Penguin Books, Ltd.

ROGOFF, Hanya. 1988. Comunicación personal.

ROGOFF, Mary. 1983. Comunicación personal.

SAMADUROFF, David. 1983. Comunicación personal citada en Jesús Rangel, "Russian Emigre Group Fading in Mexico", San Diego Union, junio 20 de 1983 , pp. B-1 y 2 .

SCHMIEDER, Oscar. 1928. "The Russian Colony of Guadalupe Valley". Lower California Studies II. University of California Publications in Geography 2 (14). University of California Press, Berkeley.

SHUBIN, Peter Phillip. 1963. Documento inédito, aún sin título. Molokan History. Los Angeles.

SOKOLOFF, Lilian. 1918. "The Russians in Los Angeles". Sociological Monographs. University of Southern California Press, Los Angeles.

STEPNIAK, A., K. A. Kravchinskii y Sergei Mikhailovich. 1888. The Russian Peasantry. Harper and Brothers, New York.

STORY, Sindey Rochelle. 1960. Spiritual Christians in Mexico: Profile of a Russian Village. Tesis para obtener el grado de doctor en antropología. University of California, Los Angeles.

STRUVE, Nikita. 1967. Christians in Contemporary Russia. Scribner's Sons, New York.

TOLSTOI, L.N. 1933. Polnoe sochranie sochinenii, vol. 72.

WILSON, Bryan R. 1970. Religious Sects. Oxford University Press. London.

YOUNG, Pauline. 1927. "Family Organization of the Molokan: A Study in Primary Group Relations". Sociology and Social Research XII (1). 1932. The Pilgrims of Russian-Town: The Community of Spiritual Christian Jumpers in America. The Struggle of a Primitive Religious Society to Maintain Itself in an Urban Environment. University of Chicago Press, Chicago.

Periódicos San Diego Ĺnion de:

26 de agosto 1905, p. I, col. 6.

5 de septiembre de 1905 , p. 7 , col. 6 .

12 de julio de 1958 , p. 1 , col. 4.

13 de julio de 1958, p. 1, cols. 4-5.

14 de julio de 1958, p. 1, cols. 6-7.

15 de julio de 1958 , p. 5, cols. 1-2.

8 de agosto de 1958, p. 5, col. 1.

11 de julio de 1959 , p. 5 , col. 1. 